\title{
Consumo de vasijas cerámicas en un contexto público tardío del Pucara de Volcán (Dto. Tumbaya, Jujuy, Argentina)
}

\author{
María Beatriz Cremonte* \\ Agustina Scaro*
}

\begin{abstract}
CREMONTE, M.B.; SCARO, A. Consumo de vasijas cerámicas en un contexto público tardio del Pucara de Volcán (Dto. Tumbaya, Jujuy, Argentina). Revista do Museu de Arqueologia e Etnologia, São Paulo, 20: 147-161, 2010.
\end{abstract}

Resumo: Neste trabalho se analisam as cerâmicas escavadas em dois recintos e uma lixeira do Pucara de Volcán e se estabelecem hipóteses sobre o consumo diferencial da cerâmica neste contexto Inca local da Quebrada de Humahuaca. O Pulcara de Volcán é um assentamento conglomerado de aproximadamente 7 hectares, ocupado com maior intensidade durante os Periodos dos Desenvolvimentos Regionais (Intermediário Tardio) e Inca. O recinto R5 está situado no setor ocidental do assentamento, diretamente associado ao recinto R2, à lixeira Tum1B2 e a um complexo de praça- montículo artificial -cemitério. Os três contextos cerâmicos estudados indicam um consumo em nivel supradoméstico: R5 como lugar de preparação de alimentos a ser consumidos em eventos públicos, Tum1B2 como área de descarte do vasilhame de serviço e R2 como provável depósito de grandes vasilhas.

Palavras-chave: Noroeste da Argentina - Quebrada de Humahuaca Pucara de Volcán - Consumo de cerâmica.

\section{El consumo de vasijas}

- $n$ los últimos años los estudios sobre

E el consumo a nivel doméstico o supradoméstico de alfarerias ha cobrado mayor importancia (Douglas e Isherwood 1990; Miller 1995a y b; Hodder 1982; Costin y Earle 1989). Como sustento de la línea metodológica aplicada en este trabajo hemos considerado válidos los conceptos definidos por Bugliani

$\left.{ }^{*}\right)$ CONICET. Instituto de geologia y Mineria, Universidad Nacional de Jujuy (IDGYM-FHyCS) <cremontebeatriz@gmail.com>, <teye29@hotmail.com>
(2006) y Bourdieu (1979). Estos autores concuerdan en que el consumo de vasijas cerámicas es un proceso por medio del cual los agentes sociales hacen estables y visibles categorias de una cultura, estableciendo espacios de diferencia social y de distinción simbólica. En este sentido, se debe considerar la manera en que los agentes sociales inscriben sus experiencias diferenciales en los bienes a partir de su posición en el espacio social.

En sociedades estatales, tales como el Imperio Inca, el consumo de bienes se enmarca en criterios económicos, sociales y principalmente politicos. Estos últimos se relacionan con dinámicas de control y legitimación, en las 
que el acceso diferencial a ciertos bienes permite controlar a la población y legitimar el poder centralizado y consolidado (Costin y Earle 1989; Bray 2003). En este trabajo, hemos intentado identificar un caso de consumo diferencial de vajilla que se vincularia con eventos públicos desarrollados en un sector del Pucara de Volcán, durante la época de anexión de este asentamiento al incario. El registro de las localizaciones de los hallazgos, sumado a la clasificación morfológica y estilistica de las vasijas permiten plantear un área de procesamiento, preparación y almacenaje transitorio de alimentos en el recinto R5 (en adelante R5); otro sector probablemente destinado a depósito de vasijas grandes, como el recinto R2 (en adelante R2) y un lugar restringido de descarte planificado, fundamentalmente de vajilla de servicio en el basurero Tum1B2. Las dataciones cronométricas obtenidas de R5 y de Tum1B2 resultaron ser sincrónicas y refieren a prácticas sociales realizadas en relación con el complejo de plaza- montículo artificial-cementerio durante el lapso de dominación Inca.

La cerámica del Pucara de Volcán perteneciente a los contextos tardios estudiados se inscribe, aunque con algunas variaciones locales de manufactura (Cremonte y Solis 1998; Cremonte 2006), al estilo regional Negro sobre Rojo característico de la Quebrada de Humahuaca, asimismo se asocia a otros tipos locales y no locales. La cerámica tardia de la Quebrada de Humahuaca se caracteriza por una profusión de vasijas con motivos geométricos en negro sobre engobe rojo, fechadas desde ca. 1.280 d.C. (Nielsen 1997) hasta los fines de la época prehispánica y aún hasta la Colonial temprana. En su gran mayoria los motivos pintados representan bandas reticuladas, triángulos en negro sólido o reticulados, espirales, "manos" o "alas", cruces, dameros, etc., organizados en diferentes pero no muy variadas configuraciones. Estas configuraciones pueden reiterarse en el interior de cuencos (pucos) o escudillas, en el exterior y bordes internos de cántaros, tinajas u ollas.

Además de las cerámicas lisas engobadas y de las Ordinarias, otros tipos/estilos asociados son los pucos Poma Negro sobre Rojo, decora- dos externamente con bandas gruesas curvilineas; los pucos con interior negro pulido y las vasijas Angosto Chico Inciso que presentan hileras de puntos incisos en sus cuellos. Directamente vinculadas al momento incaico, a estas cerámicas se asocian vasijas corrugadas, pucos de superficies bruñidas rojas, negras, marrones o combinadas (Cremonte y Botto 2009) y vasijas pequeñas Yavi-Chicha e Inca-Paya. Durante la época de dominación Inca se hacen más frecuentes en las alfarerias locales las superficies pulidas y los diseños pintados en trazo fino.

\section{El sector occidental del Pucara de Volcán}

El Pucara de Volcán es un poblado tardio de carácter conglomerado que se extiende por más de siete hectáreas sobre un cono suspendido a $2.070 \mathrm{msnm}$, ubicado de manera transversal al eje del rio Grande ( $150 \mathrm{~m}$ por sobre el fondo de valle) y a $2 \mathrm{~km}$ del actual poblado de Volcán (Fig. 1). El sitio se ubica en el limite entre la Prepuna y las Yungas empobrecidas: esta posición ecotonal, además del fácil acceso a la Puna y a los valles orientales, habria sido estratégica permitiendo la rápida obtención de bienes complementarios (Garay de Fumagalli 1998).

Las posibilidades óptimas para la ocupación humana que presenta el Pucara de Volcán fueron aprovechadas de manera continua desde el siglo XIII hasta por lo menos el siglo XVI (Garay de Fumagalli y Cremonte 1997). Una ocupación anterior hallada en el sitio por debajo del Basurero Tum1B3 fue fechada a inicios de la Era Cristiana, ella estaba separada de la ocupación más tardía por un silencio arqueológico de más de 1.000 años y se corresponde con la entidad cultural San Francisco del periodo Formativo (Garay de Fumagalli y Cremonte 2002). Otra ocupación, intermedia, tentativamente ubicada entre los siglos XI y XII, es señalada por unas vasijas descontextualizadas asignables al estilo Isla y que hoy en dia forman parte de la colección depositada en el Museo "Eduardo Casanova" de Tilcara y a dos vasos kero de oro excavados por Gatto en el Yacimiento 10 (Gatto 1946). 


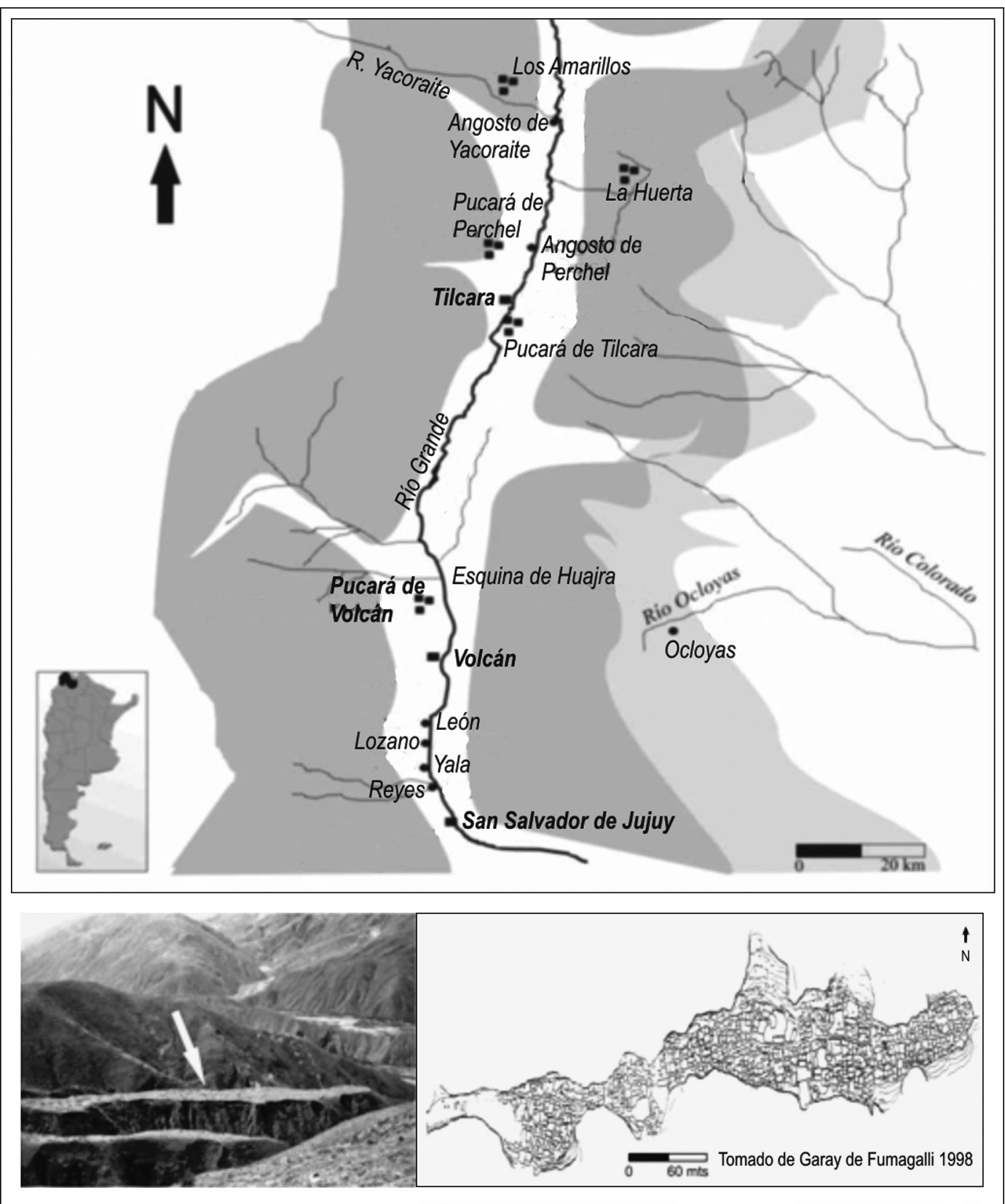

Fig. 1. Ubicación del Pucara de Volcán en la Quebrada de Humahuaca. Vista general del sitio y plano.

Recientemente, el hallazgo de material Isla en un sitio ubicado en la quebrada de Huajra (aproximadamente $5 \mathrm{~km}$ al norte del Pucara de Volcán), permite comenzar a contextualizar y delinear la presencia y circulación de este estilo en el sector sur de la Quebrada de Humahuaca (Cremonte et al. 2009).
El Pucara de Volcán se compone de unos 600 recintos contiguos de diversos tamaños (Fig. 1) que forman agrupaciones delimitadas por caminos y presentan homogeneidad a nivel arquitectónico. Se trata de recintos rectangulares con sus ángulos redondeados, de muros dobles rellenos con guijarros y barro batido. El 
conjunto se completa con un camino axial sobreelevado que divide longitudinalmente al sitio en dos mitades, del mismo se desprenden caminos secundarios también sobreelevados que recorren todo el asentamiento.

En el extremo occidental del Pucara se extiende un gran espacio abierto, se trataria de una plaza de aproximadamente $2.400 \mathrm{~m}^{2}$ asociada a un montículo artificial que habria contenido una tumba en su cima (saqueada antes de realizarse las primeras excavaciones sistemáticas en el sitio) y a un área de entierros en cámaras circulares de piedra (Fig. 2). Con respecto a los enterratorios, el sitio presenta además cámaras mortuorias también segregadas del área nuclear residencial, ubicadas al noroeste de la misma, sobre otro cono de deyección separado por un surco de escurrimiento (Garay de Fumagalli 1998). Este complejo de plaza- monticulo artificial-cementerio, hallado en distintos sitios tardios del área surandina, refleja el uso y significación simbólica de un espacio que se vincularía con rituales y ceremonias comunitarias (Nielsen 2006). Espacios públicos como los señalados serian una expresión del aparato administrativo que demostraria el control a través del cual se dirigia a la fuerza de trabajo hacia la producción estatal, rasgo que se reitera durante el incario. Además, el conjunto de plaza-monticulo-cementerio y el camino axial sobreelevado serian "remodelaciones" realizadas por la administración Inca en el poblado preexistente, pudiendo albergar a una población más numerosa a partir de la primera mitad del siglo $\mathrm{XV}$, a juzgar por los fechados obtenidos de los grandes basureros Tum1B1 y Tum1B3 emplazados en el área residencial (Garay de Fumagalli y Cremonte 1997) correspondientes a los niveles que presentan cerámica Inca local y Fase Inca.

En el Pucara de Volcán el complejo de plaza-montículo-cementerio se asocia espacialmente al pequeño basurero HumahuacaInca Tum1B2 y a un conjunto de recintos, de los cuales fueron excavados dos, R5 y R2. Las dataciones cronométricas realizadas sobre carbón vegetal ubican al basurero Tum1B2 en el $440 \pm 60$ AP (calibr. 1d: 1410
- $1520 A D / 1600$ - $1620 A D$; calibr. 2d: 1390 - $1640 A D)$, mientras que al piso de R5 en el $435 \pm 50$ AP (calibr. 1d: $1410-$ $1500 \mathrm{AD} / 1600$ - $1620 \mathrm{AD}$; calibr. 2d: 1400 - $1530 \mathrm{AD} / 1550$ - $1640 \mathrm{AD}) .{ }^{1}$ Estos fechados indican contemporaneidad entre la época de formación del basurero Tum1B2 y el piso de R5, ambos asignables al momento Inca. ${ }^{2}$ En las estructuras excavadas el material predominante es la cerámica debido a las pobres condiciones de conservación de elementos tales como madera, calabaza o hueso imperantes en el Pucara de Volcán. Por otro lado, la escasez de artefactos y desechos líticos en las estructuras excavadas es una situación recurrente en otros sitios del Periodo Inca del Noroeste Argentino. Otros recintos excavados en el Pucara de Volcán (R1, R3 y R4) se encuentran en áreas residenciales distantes de este sector, revelan contextos domésticos sin evidencias de una filiación incaica, y además carecen de dataciones cronométricas hasta el momento, razones por las cuales no fueron incluidos en este estudio.

En relación con el impacto o vínculo entre el Inca y las poblaciones locales, es sabido que en el uso y control de los nuevos territorios, la administración Inca construyó asentamientos tanto en lugares poblados como en zonas vacias. También se ha planteado que el sistema de administración pudo desarrollarse a través de una serie de estrategias para consolidar el control sobre los grupos sometidos, considerando al control territorial y al control hegemónico (Hassig 1985; Luttwak 1976) como extremos de un espectro de situaciones (Cremonte y Williams 2007).

El paisaje social construido en el sur de la Quebrada de Humahuaca y su borde oriental durante la época de dominación Inca parece ser una combinación entre ambos tipos de controles. En el Pucara de Volcán las evidencias de un

(1) Los fechados fueron calibrados con el programa Oxcal 3.9 (Ramsey, C. 2002)

(2) Del recinto R2 no se obtuvieron materiales para dataciones cronométricas pero la cerámica es asignable al momento Inca. 


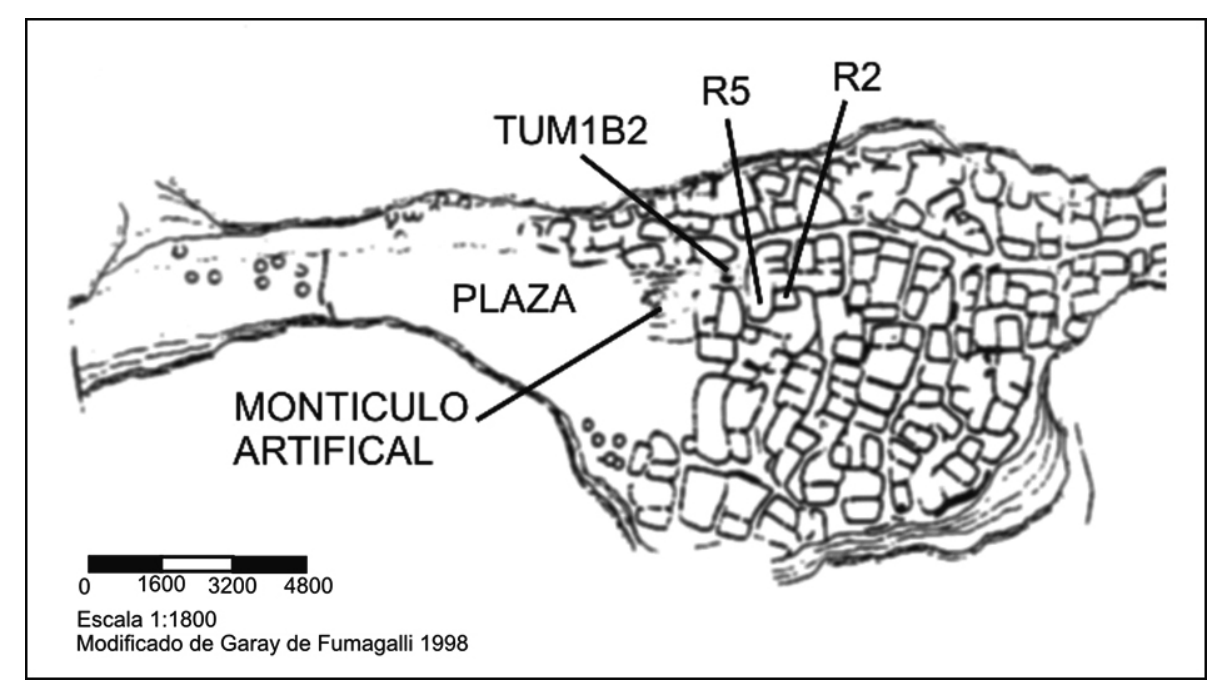

Fig. 2. Sector occidental del Pucara de Volcán (Quebrada de Humahuaca, Jujuy).

control hegemónico se materializarian precisamente en ese complejo de plaza-montículo artificial-cementerio donde, a través de ceremonias y festividades se pactaría la afiliación al incario de la población local y la disponibilidad de una numerosa mano de obra para la mit'a. (Cremonte y Williams 2007).

Por otro lado, y como ha sido señalado recientemente, en el marco de las diversas situaciones locales y de las estrategias e intereses del Inca, deben evaluarse patrones de cambio a nivel doméstico, tales como hábitos alimentarios, condiciones de salud e intensificación de la producción (Santoro et al. 2010). Respecto de lo último, próximo al Pucara de Volcán y al sitio incaico Esquina de Huajra (ubicado $5 \mathrm{~km}$ al norte del Pucara de Volcán) se ubica el área agricola de Raya Raya con una superficie aproximada de 60 hectáreas y que presenta técnicas constructivas incaicas. Raya Raya así como zonas agricolas cercanas pero menores se suma a otras áreas de cultivo de Jujuy y del Noroeste Argentino que indican que la intensificación agrícola fue uno de los objetivos de la economía politica Inca. Además, en Raya Raya pudo ser importante la producción de maiz, ya que los análisis isotópicos de restos óseos humanos de Esquina de Huajra indicaron un importante consumo de plantas $\mathrm{C}_{4}$ (que para la zona corresponde al maiz) combinado con consumo de carne (Williams et al. 2005).
Los contextos cerámicos estudiados

Las formas, distribución y tamaños de los contenedores cerámicos usados para preparar, almacenar y servir alimentos permiten establecer pautas de consumo diferencial de la alfareria, en distintos contextos de asociación (domésticos, mortuorios, públicos) o en diferentes localizaciones dentro de un mismo contexto. A continuación se caracterizan los conjuntos alfareros recuperados en cada una de las estructuras excavadas. Dicha caracterización se realizó considerando el Número Minimo de Vasijas (NMV) de servicio, así como para la preparación y almacenaje de alimentos; los tipos y estilos presentes y las asociaciones espaciales de los contenedores con otros elementos del registro arqueológico.

\section{El recinto $\mathrm{R} 5$}

Se trata de un recinto rectangular de ángulos redondeados de $40 \mathrm{~m}^{2}$ de superficie (Fig. 2), cuya excavación parcial (80\%) reveló un único piso de ocupación entre los 65 y 82 $\mathrm{cm}$ de profundidad. El piso de ocupación de R5 (Fig. 3) corresponde a un sedimento limo arenoso que presenta manchas de carbón. En el mismo se halló un rasgo negativo, un pozo de $30 \mathrm{~cm}$ de diámetro y $45 \mathrm{~cm}$ de profundidad 
recubierto con lajas, que probablemente correspondería a un poste para sostener el techo de la estructura.

Del material cerámico recuperado (Tabla 1) se identificaron veintidós vasijas fragmentadas correspondientes a cinco ollas ordinarias, seis ollas y un cántaro Angosto Chico Inciso, cinco vasijas restringidas Humahuaca Negro sobre Rojo, dos pucos Bruñidos, un vasito ordinario, una tapa de olla reciclada a partir de una escudilla Humahuaca Negro sobre Rojo y un puco Inca-Paya.

Las vasijas ordinarias son en su mayoría piezas restringidas correspondientes a ollas, cántaros y tinajas ${ }^{3}$ de diversos tamaños, que presentan en algunos casos hollin en la superficie externa. El grupo de vasijas ordinarias se completa con un vasito de color castaño. Las ollas Angosto Chico Inciso son de tamaño mediano a grande y se caracterizan por tener incisiones irregulares arrastradas horizontales en el área del cuello. El pequeño cántaro de este estilo tiene incisiones punteadas subtriangulares.
Las vasijas Humahuaca Negro sobre Rojo tienen superficies pulidas y alisadas. Los motivos decorados corresponden a reticulados en línea fina, semicírculos concéntricos ubicados en los bordes internos de las vasijas cerradas, bandas reticuladas asociadas a triángulos negros sólidos y banderines reticulados. Estas vasijas presentan hollin en sus superficies externas aunque no se hallaron fogones en el interior del recinto, indicando tal vez que existiria una estructura de combustión en un sector externo a R5 o bien el traslado de los recipientes desde probables unidades domésticas.

La alfarería analizada de R5 muestra predominio de vasijas grandes restringidas, probablemente empleadas en el procesamiento y almacenaje de alimentos, mientras que la vajilla de servicio (pucos, platos y escudillas) es muy escasa. Estas grandes vasijas cerradas se ubicaban preferentemente junto a los muros sur y oeste del recinto, indicando que en estos sectores probablemente se concentrarian las actividades de preparación y almacenaje de alimentos. Junto al muro sur, los fragmentos

Tabla 1

\begin{tabular}{ccc}
\hline & Vasijas Cerámicas del recinto R5 & \\
\hline \hline & Número Mínimo de Vasijas : 22 & \\
\hline Función & Formas & Tipos cerámicos \\
\hline Tinaja: 3 & Olla: 12 & Humahuaca Negro sobre Rojo \\
Preparación-Almacenaje & & Angosto Chico Inciso \\
de alimentos N. M. V.: 17 & Humahuca Negro sobre Rojo & Ordinario Alisado \\
& Cántaro: 1 & Angosto Chico Inciso \\
& No Determinable: 1 & Humahuca Negro sobre Rojo \\
\hline Servicio N. M. V: 4 & Puco: 3 & Inca-Paya \\
& & Bruñido \\
& Vaso: 1 & Ordinario \\
\hline
\end{tabular}

1 base reutilizada de escudilla Humahuaca Negro sobre Rojo

(3) Basándonos en Bugliani (2006): Olla es un recipiente poco restringido con bordes evertidos y cuello corto o sin él. Cántaro es una pieza cerrada muy restringida respecto del diámetro máximo, con cuellos estrecho y alto; el diámetro minimo es superior o igual a un tercio del diámetro máximo. Tinaja es un recipiente cerrado restringido a poco restringido con cuello corto muy evertido. 


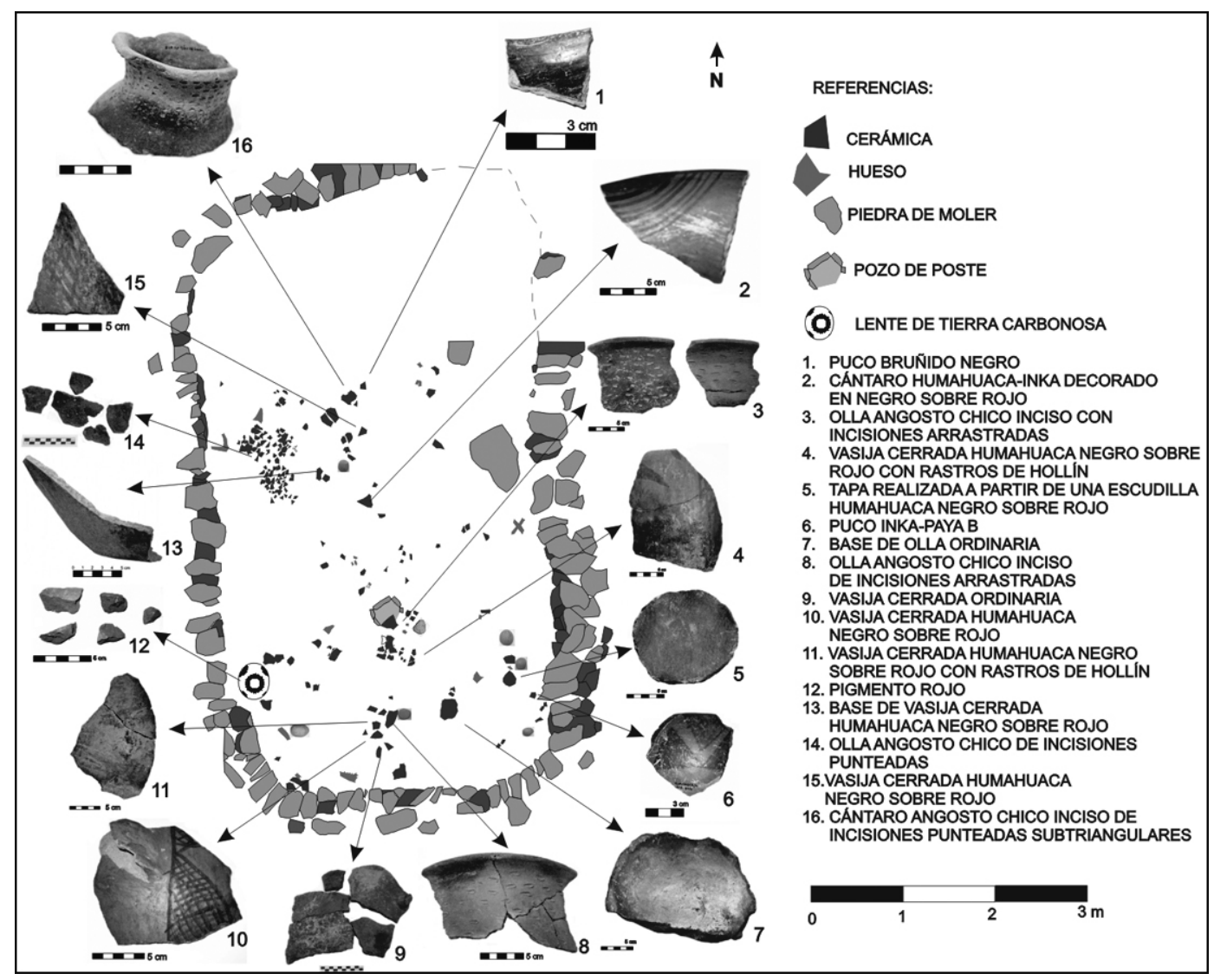

Fig. 3. Planta del piso del Recinto R5 con la ubicación de los hallazgos.

cerámicos se hallaron asociados a seis manos de moler, escasos restos óseos y restos de pigmento rojo (arcilla con abundante óxido ferroso), mientras que junto al muro oeste, la cerámica se vinculaba espacialmente con restos óseos faunísticos. Otra área de actividad se ubicaría en el sector este del recinto, en el que se encontraron dos grandes piedras de moler sin fragmentos cerámicos asociados; este sector probablemente estuvo destinado a la molienda.

Además de actividades de molienda, preparación y almacenaje de alimentos, es muy probable que en R5 se hayan llevado a cabo otras actividades, tal vez de menor importancia. Así lo indica el hallazgo de esquirlas de obsidiana, débil evidencia de manufactura o tal vez de mantenimiento de artefactos líticos, y del mencionado pigmento rojo, quizás usado en la terminación o decoración de objetos. Estas evidencias permiten plantear diferentes acciones que podrian ser tentativamente vinculadas a producciones de carácter multi-artesanal (Shimada 2007).

\section{El recinto $\mathrm{R} 2$}

Este recinto se encuentra adosado a $\mathrm{R} 5$ y es de menor tamaño: tiene $25 \mathrm{~m}^{2}$ de superficie (Fig. 2). Se trata también de una estructura rectangular con sus cuatro ángulos redondeados, presentando como única diferencia una mayor regularidad en la construcción de los muros. El piso de ocupación de R2 fue hallado entre $75 \mathrm{y}$ $80 \mathrm{~cm}$ de profundidad y corresponde a un sedimento limo arenoso con arcilla. Al igual que en $\mathrm{R} 5$, en este recinto los fogones también están ausentes. A partir de los fragmentos recuperados en el piso de ocupación (Fig. 4), se identificaron dieciocho vasijas (Tabla 2) que corresponden a dos ollas medianas Angosto 
Chico Inciso, tres ollas y un puco ordinarios; dos cántaros, dos ollas y un plato todos decorados en Negro sobre Rojo; cuatro vasijas pulidas de color morado, castaño y rosado, dos pucos con interior negro pulido y un puco Poma Negro sobre Rojo. Los únicos elementos no cerámicos encontrados en este recinto corresponden a algunos fragmentos óseos de camélidos y a cuatro piedras planas (lajas) probablemente utilizadas como superficies de apoyo. A diferencia de R5 no es posible plantear asociaciones directas entre los hallazgos.

Las vasijas Humahuaca Negro sobre Rojo halladas en este recinto están decoradas con triángulos reticulados, espirales reticuladas y dos bandas reticuladas separados por una linea negra gruesa. Las vasijas cerradas presentan además semicírculos concéntricos en los bordes internos. A diferencia de las piezas de este estilo halladas en R5, las de R2 no exhiben hollin. La única vasija Humahuaca-Inca de este recinto corresponde a una escudilla decorada en negro sobre engobe rojo pulido que presenta un espiral reticulado en linea fina.

Por otra parte, las ollas Angosto Chico Inciso, presentes en un número comparativa- mente menor al de R5, son más pequeñas y de paredes más delgadas, decoradas en la mayoría de los casos con incisiones punteadas.

En R2 tampoco está representada la vajilla de servicio y, si tenemos en cuenta la cantidad de ollas y tinajas en relación con la superficie excavada, vemos que hay mayor concentración de vasijas que en R5, pudiendo ser un depósito directamente vinculado a este último.

\section{El basurero Tum1B2}

El pequeño basurero Tum1B2 (Fig. 2), fechado en el $440 \pm 60 \mathrm{AP}$, refleja un consumo cerámico diferente al de R2 y R5, así como de los otros basureros excavados ubicados en el centro del área residencial del Pucara de Volcán: Tum1B1 y Tum1B3 (Cremonte y Nieva 2003). Este basurero tiene $90 \mathrm{~cm}$ de potencia y es muy homogéneo en cuanto a la composición de los materiales cerámicos analizados.

A partir de los casi 500 fragmentos hallados en Tum1B2 se identificaron 70 recipientes (Tabla 3), de los cuales el 60\% corresponde a vajilla de servicio: pucos, escudillas, platos,

Tabla 2

\begin{tabular}{|c|c|c|}
\hline \multicolumn{3}{|c|}{ Vasijas Cerámicas del recinto $\mathrm{R} 2$} \\
\hline \multicolumn{3}{|c|}{ Número Mínimo de Vasijas : 18} \\
\hline Función & Formas & Tipos cerámicos \\
\hline \multirow{4}{*}{$\begin{array}{l}\text { Preparación-Almacenaje } \\
\text { de alimentos N. M. V.: } 13\end{array}$} & Tinaja: 2 & $\begin{array}{l}\text { Ordinario } \\
\text { Pulido Liso }\end{array}$ \\
\hline & Olla: 5 & $\begin{array}{c}\text { Angosto Chico Inciso } \\
\text { Ordinaria Naranja Alisada } \\
\text { Pulido Liso } \\
\text { Humahuaca Negro sobre Rojo }\end{array}$ \\
\hline & Cántaro: 2 & Humahuaca Negro sobre Rojo \\
\hline & No Determinable: 4 & $\begin{array}{c}\text { Ordinario } \\
\text { Humahuaca Negro sobre Rojo } \\
\text { Pulido Liso }\end{array}$ \\
\hline \multirow[t]{2}{*}{ Servicio N. M. V: 5} & Puco: 4 & $\begin{array}{c}\text { Interior Negro Pulido } \\
\text { Ordinario Naranja Alisado } \\
\text { Puco Poma }\end{array}$ \\
\hline & Escudilla: 1 & Humahuaca-Inca \\
\hline
\end{tabular}




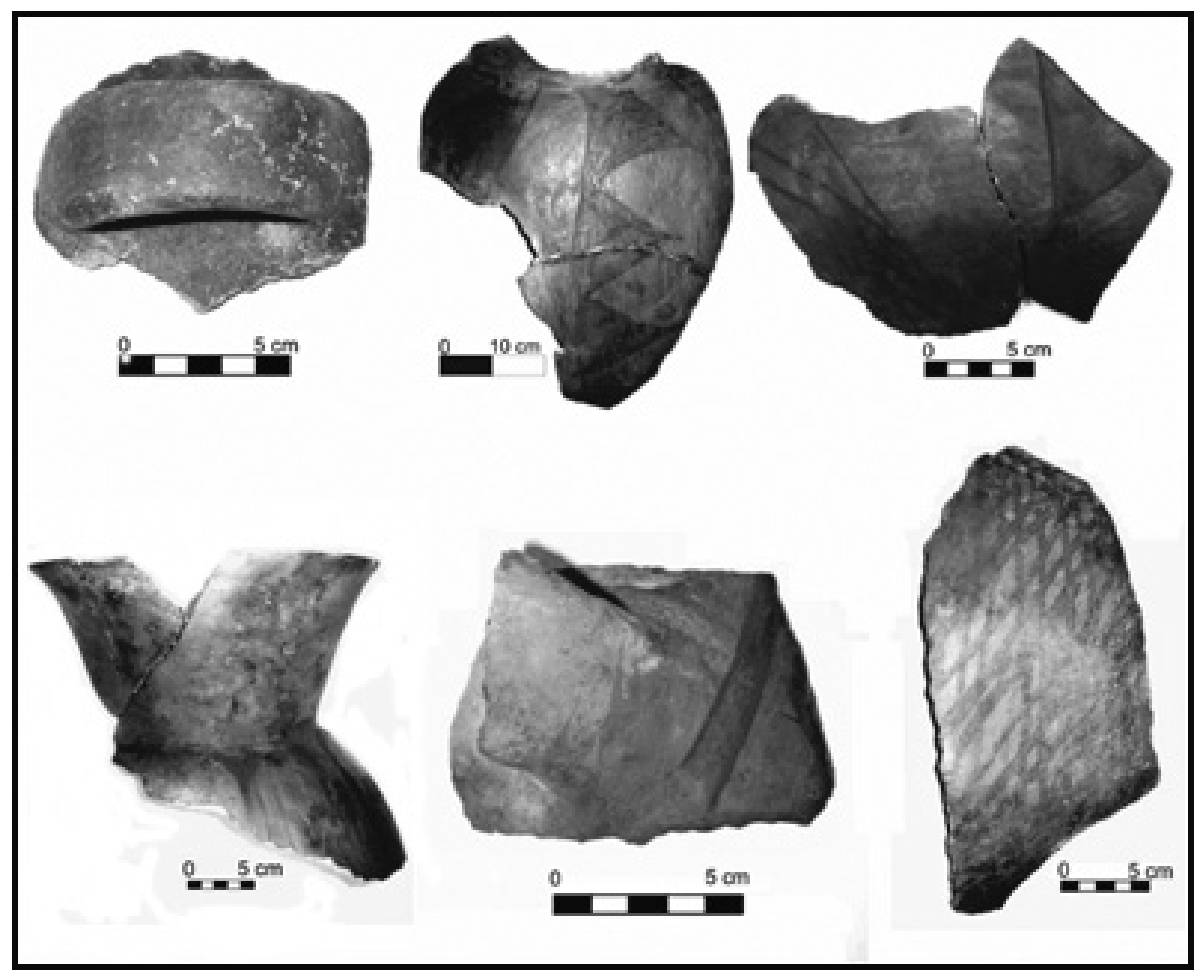

Fig. 4. Fragmentos representativos de las vasijas grandes procedentes del Recinto R2.

tazones, fuentes y baldes. ${ }^{4} \mathrm{Si}$ consideramos el predominio de superficies pulidas y diseños de trazo fino, es posible proponer que esta vajilla seria de uso preferencial (es decir vasijas priorizadas en relación a otras). En la Figura 5 se muestran algunos de los fragmentos más representativos de Tum1B2. El conjunto hallado en este basurero incluye asimismo 29 vasijas cerradas (tinajas, ollas, cántaros y aribalos) ordinarias y de los estilos HumahuacaInca e Inca-Paya.

De este conjunto cerámico se destaca la mayor proporción de vasijas abiertas en relación a las cerradas, así como también la presencia de vasijas con superficies pulidas y decoradas con trazos finos, un rasgo caracteristico de la cerámica local durante el incaico en la región.

(4) Un balde es un recipiente cuyo diámetro de abertura no es menor al $80 \%$ del diámetro máximo (Ortiz y Delgado, 1997).

\section{Conclusiones: el consumo cerámico en un contexto público}

Los contextos cerámicos analizados para R5 y R2 y para el basurero Tum1B2 serian aparentemente funcionales a las actividades desarrolladas en el espacio de participación comunitaria cercana, el complejo de plaza- monticulo artificial-cementerio (Fig. 2). Estos tres contextos son asignables al momento Inca e indicarian un consumo a nivel supradoméstico. Las actividades aludidas corresponderían a eventos públicos o teatralizaciones rituales, entendiendo por las mismas a representaciones no cotidianas, organizadas y dirigidas por agentes politicos en épocas y localizaciones especificas y que incluian elementos de teatralización, invocaciones cosmológicas y fiestas (Coben 2006).

En principio, los recintos analizados poseen conjuntos cerámicos que marcan pautas de consumo diferencial en relación a las formas y diseños de las vasijas, permitiendo aproximarnos a las actividades que habrian tenido lugar en el interior de los mismos. 
Tabla 3

\begin{tabular}{|c|c|c|}
\hline \multicolumn{3}{|c|}{ Vasijas Cerámicas del basurero Tum1B2 } \\
\hline \multicolumn{3}{|c|}{ Número Mínimo de Vasijas : 70} \\
\hline Función & Formas & Tipos cerámicos \\
\hline \multirow[t]{2}{*}{$\begin{array}{l}\text { Preparación-Almacenaje } \\
\text { de alimentos N. M. V.: } 27\end{array}$} & Tinaja: 7 & $\begin{array}{c}\text { Ordinario } \\
\text { Alisado con engobe rosado } \\
\text { Inca-Paya } \\
\text { Humahuaca Negro sobre Rojo }\end{array}$ \\
\hline & Olla: 20 & $\begin{array}{c}\text { Angosto Chico Inciso } \\
\text { Ordinario }\end{array}$ \\
\hline \multirow{7}{*}{ Servicio N. M. V: 45} & Puco: 28 & $\begin{array}{c}\text { Humahuaca-Inca } \\
\text { Interior Negro Pulido } \\
\text { Bruñido } \\
\text { Borravino sobre Naranja } \\
\text { Poma Pomeño } \\
\text { Pulido Liso (castaño, rojo) }\end{array}$ \\
\hline & Escudilla: 12 & $\begin{array}{c}\text { Portillo Policromo (Yavi-Chicha) } \\
\text { Portillo Morado (Yavi-Chicha) } \\
\text { Humahuaca-Inca } \\
\text { Marleado } \\
\text { Alisado con engobe } \\
\text { Pulido Liso }\end{array}$ \\
\hline & Aribalo: 2 & $\begin{array}{c}\text { Humahuaca-Inca } \\
\text { Pulido Liso (castaño) }\end{array}$ \\
\hline & Plato: 2 & Humahuaca-Inca \\
\hline & Fuente: 1 & Alisado con engobe rojo \\
\hline & Balde: 1 & Humahuaca Negro sobre Rojo \\
\hline & Tazón: 1 & Alisado con engobe rojo \\
\hline
\end{tabular}

En R5 predominan las vasijas grandes y medianas destinadas a la preparación y el almacenaje, de las cuales la mayoria corresponden a ollas del estilo Angosto Chico Inciso que presentan una mayor variedad en el tipo de incisiones respecto de R2. Las piezas más grandes fueron decoradas con incisiones más profundas e irregulares y los bordes son de labios gruesos y evertidos. En R2 las ollas de este estilo son medianas y fueron decoradas con incisiones del tipo punteado subtriangular. Estas ollas son más pequeñas y de mejor terminación que las observadas en R5.

En ambos recintos, las ollas Angosto Chico
Inciso están asociadas a cántaros y tinajas decorados con motivos geométricos en negro sobre fondo rojo. Los motivos predominantes son paneles verticales reticulados en linea fina y media y semicírculos concéntricos en el interior de cuellos evertidos. En R5 las vasijas decoradas presentan rastros de hollin, mientras que las de R2 no lo tienen.

En contraste con la variedad de vasijas mencionadas para preparación-almacenaje (cántaros, tinajas y ollas), vemos que en ambos recintos las vasijas de servicio (pucos, platos, escudillas) están prácticamente ausentes (Fig. 6). Las últimas se limitan a dos pucos Bruñidos, 


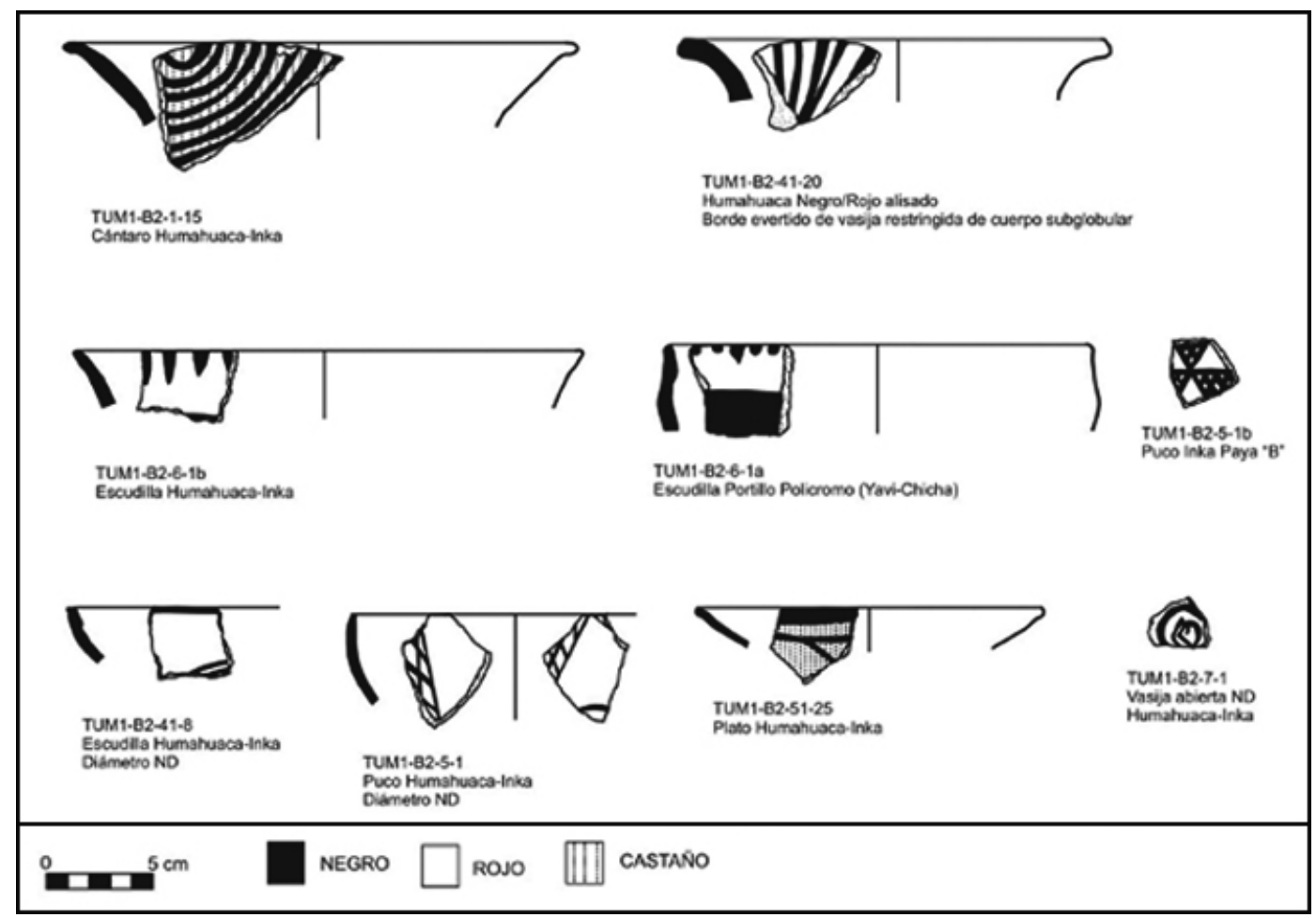

Fig. 5. Fragmentos de la vajilla de servicio procedente del basurero Tum1 B2.

un puco Poma Negro sobre Rojo, una escudilla HumahuacaInca, dos pucos con interior negro pulido y un puco Inca-Paya.

Sobre la base de las

diferencias mencionadas, proponemos que $\mathrm{R} 5$ reflejaría actividades involucradas con el procesamiento (en relación con los instrumentos de molienda), la preparación y almacenaje de alimentos, aunque no con la cocción de los mismos ya que no fueron hallados fogones ni otros rastros de combustión. Es probable que en este recinto se llevaran a cabo algunos pasos del proceso de elaboración de

Fig. 6. Porcentajes comparativos de tipos funcionales de vasijas procedentes de los recintos

R5, R2 y basurero Tum1 B2.

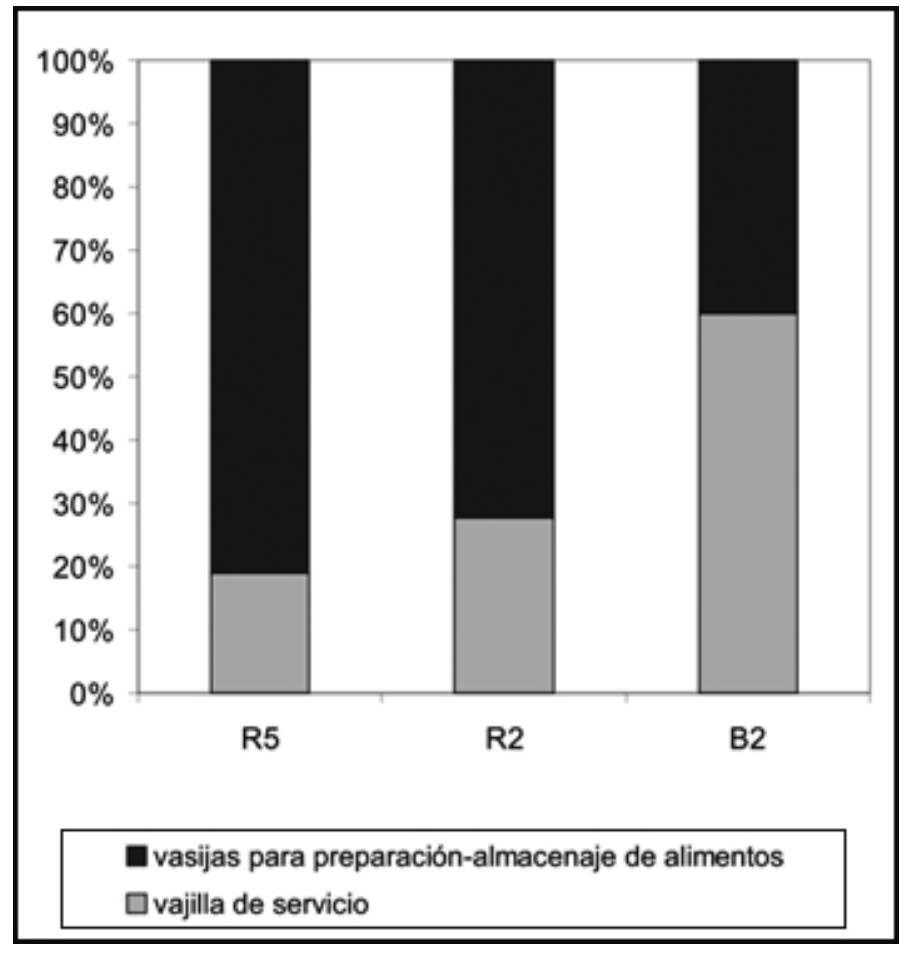


chicha, tales como el molido del maiz, la fermentación de la "chuya" en cántaros y su almacenaje temporario en grandes vasijas (Cremonte et al. 2009), por ejemplo en algunas ollas Angosto Chico Inciso y la Ordinaria Alisada. Con respecto a utensilios comunes usados en la elaboración de la chicha, tales como cuencos de calabaza y cucharas de madera, si bien están ausentes en el registro arqueológico, ello puede deberse a las pobres condiciones de conservación imperantes. Por otra parte, la ausencia de vasijas muy grandes de boca ancha (tipo virque) en R5, podria estar indicando que las etapas de "apuñamiento" y/o "meceado" en la preparación de esta bebida no se habrian realizado alli. Sin embargo, no debe descartarse el probable uso de vasijas de boca ancha más pequeñas, tal vez algunas de las "ollas" del estilo Angosto Chico Inciso. También es posible pensar en una situación similar a la propuesta por Moore (1989) para el sitio Chimú de Manchan, donde el equipo para fabricar chicha estaria disponible en cada unidad doméstica. Es decir que para los eventos públicos esta bebida sería producida y provista de manera autónoma por diferentes unidades domésticas, con escasa o ninguna intervención del Imperio Chimú.

En R2 se observa una concentración mayor de vasijas que en $\mathrm{R} 5$ (18 vasijas en $25 \mathrm{~m}^{2}$ ) y también están ausentes los fogones. Al igual que en $\mathrm{R} 5$, los recipientes corresponden en su mayoria a ollas, cántaros y tinajas, mientras que las vasijas para servir (pucos, platos, escudillas) son casi inexistentes. Las características de R2 permiten plantear como hipótesis de trabajo que este recinto pudo ser un depósito de vasijas grandes. Esto último se justificaria considerando las menores dimensiones de $\mathrm{R} 2$ respecto de R5, la ausencia de estructuras de combustión y proporcionalmente la mayor cantidad de piezas de grandes dimensiones.

Por último, si consideramos que los recipientes cerámicos de servicio fueron usados en contextos de interacción social de mayor visibilidad pública que los recipientes para cocción y almacenamiento, entonces el pequeño basurero Tum1B2 es revelador. Tum1B2 muestra el descarte (aunque con muy pocos fragmentos por vasija) de ollas y tinajas grandes y medianas similares a las de R5 y R2 pero también de vajilla especial: aribalos, platos, pucos, escudillas, una fuente y un probable balde. Esta vajilla de servicio corresponde a más del $60 \%$ del conjunto recuperado (Fig. 6) y pertenece al estilo local Humahuaca-Inca con motivos pintados en negro sobre rojo de trazo fino. Asimismo e indicando un consumo de vajilla preferencial, están presentes estilos no locales (pucos Bruñidos, Inca-Paya, Yavi-Chicha, Borravino sobre Naranja). Como ya se dijo, la gran mayoria de la cerámica de Tum1B2 presenta superficies muy pulidas (Cremonte y Solis 1998).

El despliegue visual de la vajilla de servicio de Tum1B2, destinada a la distribución de alimentos, nos remite a distintas celebraciones que pudieron tener lugar en la plaza, seguramente enmarcadas en el interés por reforzar la afiliación al imperio de los pobladores del Pucara. A partir de estas celebraciones se institucionalizarian una serie de prácticas de sujeción al mismo y de negociación, como por ejemplo la distribución de la mano de obra con variados fines estatales (mit'a). Grandes vasijas como las de R5 conteniendo probablemente chicha y otros alimentos, pudieron ser trasladadas a la plaza, así como la vajilla de servicio. La probable función de R2 como un depósito de vasijas grandes nos lleva también a pensar en la existencia de otro/s depósito/s para vajilla de servicio, espacialmente vinculado/s a R5 y R2.

La existencia de sectores en los que se observa un consumo diferencial de vasijas durante el Periodo Inca como hemos planteado a lo largo de estas páginas, contribuye al conocimiento de las politica económica estatal en las provincias periféricas del imperio, siendo aun poco numerosos otros casos análogos para el Noroeste Argentino. Una excepción son los estudios realizados en Catamarca, en los departamentos de Tinogasta (Orgaz et al. 2007) y Andalgalá (Williams 1991; Williams et al. 2005).

El centro incaico Potrero Chaquiago ubicado en el bolsón de Andalgalá, posee un sector denominado Retambay, donde al igual que en el sector occidental del Pucara de 
Volcán, están presentes rasgos arquitectónicos tales como plazas y plataformas de alta relevancia desde el punto de vista administrativo, político y religioso del incario. La presencia de un alto porcentaje de alfareria de estilos Inca en el contexto cerámico de Retambay, asi como de vasijas para cocción, almacenaje y servicio en la plaza o en sectores adyacentes, permitió plantear la existencia de áreas para la preparación de alimentos a gran escala (Williams 1991; Williams et al. 2005). Esta situación es similar a la propuesta para el Pucara de Volcán, donde hemos diferenciado, en relación con los eventos públicos que se habrian llevado a cabo en la plaza, un sector de preparación y almacenaje de alimentos, reflejado especialmente en R5 con un $20 \%$ de vajilla de servicio y un $80 \%$ de vasijas destinadas a la cocción y almacenaje de alimentos. Este contexto se diferencia claramente de la alfareria representada en el basurero Tum1B2 que muestra una situación opuesta a R5 y R2 (Fig. 6), predominando en un $60 \%$ la vajilla de servicio (pucos, escudillas y platos) de estilo Inca local y de tipos no locales con superficies muy pulidas.

\section{Agradecimientos}

Los estudios realizados se inscriben en el marco de los proyectos dirigidos por Cremonte PIP-CONICET 5235, ANPCYT PICT 2007 No 01538-Préstamo BID y SECTER-UNJu C-156. Agradecemos a Mercedes Garay de Fumagalli, Luis Laguna, Anibal Villarroel, Martin Arjona, Maria Tejerina, Guillermo Demitrópulos, Elizabeth Chávez y Valeria López la colaboración en las tareas de excavación y en la elaboración de los registros e informes de campo, actividades financiadas mediante el subsidio otorgado al primero de los proyectos citados.

CREMONTE, M.B.; SCARO, A. Vessels consumption in a late public context of Pucara de Volcan (Tumbaya, Jujuy, Argentina). Revista do Museu de Arqueologia e Etnologia, São Paulo, 20: 147-161, 2010.

Abstract: In this paper potteries excavated in Structure R5 from Pucara de Volcán site are analyzed and hypotheses about their differential consumption in this local Inca context in Quebrada de Humahuaca are stated. Pucara de Volcán is an approximately 7 hectares conglomerate settlement. The occupation of the site was most important during the Regional Developments (Late Intermediate) and Inca Periods. Structure R5, situated within the western zone of the settlement, is closely associated to Structure R2, the midden Tum1B2 and to a plaza-artificial mound -cemetery complex. The three pottery contexts studied point to a supra-domestic consumption: Structure R5 as a place of food preparation to be consumed in public events, Tum1B2 as a discard area mainly for service vessels and Structure R2 as a probable big vessels store.

Keywords: Northwestern Argentina - Quebrada de Humahuaca - Pucara de Volcán - Pottery consumption. 


\section{Referencias bibliográficas}

BOURDIEU, P.

1979 Distinction. Routledge \& Kegan. London.

BRAY, T.

2003 To Dine Splendidly. Imperial Pottery, Commensal Politics, and the Inca State. In: Bray, T. (Ed.) The Archaeology and Politics of Food and Feasting in Early States and Empires. Kluwer Academic /Plenum Publishers: 93-142

BUGLIANI, M.F.

2006 Consumo y representación en el Formativo del sur de los valles Calchaquies. Tesis para acceder al grado académico de Doctora en Ciencias Naturales. Facultad de Ciencias Naturales y Museo. La Plata, Universidad Nacional de La Plata.

COBEN, L.S.

2006 Other Cuzcos: Replicated Theaters of Inka Power. In: Inomata, T.; Coben L. S. (Eds.) Archaeology of Perfomance, Theaters of Power, Community, and Politics. Oxford, AltaMira Press: 223-260.

COSTIN, C.; EARLE, T.

1989 Status Distinction and Legitimation of power as reflected in changing patterns of consumption in late prehispanic Peru. American Antiquity, 54 (4): 691-714.

\section{CREMONTE, M.B.}

2006 El estudio de la cerámica en la reconstrucción de las historias locales. El sur de la Quebrada de Humahuaca (Jujuy, Argentina) durante los Desarrollos Regionales e Incaico. Chungara, 38 (2): 239-247.

CREMONTE, M.B.; BOTTO, L.

2009 Unas vasijas especiales halladas en contextos tardios del noroeste de Argentina. Manufactura de los Pucos Bruñidos. Estudios Atacameños, 37: 63-77.

CREMONTE, M.B.; NIEVA, G.

2003 Registro y clasificación cerámica del basurero Tum1B3 del Pukara de Volcán (Dto. Tumbaya, Quebrada de Humahuaca). Cuadernos, 20: 273-391.

CREMONTE, M.B.; OTERO, C.; GHEGGI, M.S.

2009 Reflexiones sobre el consumo de chicha en épocas prehispánicas a partir de un registro actual en Perchel (Dto. Tilcara, Jujuy). Relaciones de la Sociedad Argentina de Antropologia, XXXIV: 75-102.

CREMONTE, M.B.; PERALTA, S.; SCARO, A.

2009 Primera prospección arqueológica en un camino hacia y desde las yungas (Dto. Tumbaya, Jujuy). Pacarina. En Prensa.

CREMONTE, M.B.; SOLÍS, N.

1998 La cerámica del Pucara de Volcán: variaciones locales y evidencias de interacción. In: Cremonte, M.B. (Org.) Los Desarrollos Locales y sus Territorios. Arqueología del NOA y Sur de Bolivia. San Salvador de Jujuy, Ediunju: 155-196.

CREMONTE, M.B.; WILLIAMS, V.

2007 La construcción social del paisaje durante la dominación Inka en el Noroeste Argentino. In: Nielsen, A.; Rivolta, C.; Seldes, V.; Vázquez, M.; Mercolli, P. (Comp.) Procesos Sociales prehispánicos en el sur andino. La vivienda, la comunidad y el territorio. Córdoba, Editorial Brujas: 207-236.

DOUGLAS, M.; ISHERWOOD , B.

1990 El mundo de los bienes; hacia una antropología del consumo. México: Editorial Grijalbo.

GARAY DE FUMAGALLI, M.

1998 El Pucará de Volcán, Historia Ocupacional y Patrón de Instalación. In: Cremonte, M.B. (Org.) Los Desarrollos Locales y sus Territorios. Arqueología del NOA y Sur de Bolivia. San Salvador de Jujuy, Ediunju: 131-153.

GARAY DE FUMAGALLI, M.; CREMONTE, M.B.

1997 Correlación Cronológica del Yacimiento de Volcán, con sitios de los Valles Orientales (Sector Meridional, Quebrada de Humahuaca). Avances en Arqueología, 3: 191-212.

GARAY DE FUMAGALLI, M.; CREMONTE, M.B. 2002 Ocupaciones agropastoriles tempranas al sur de la Quebrada de Humahuaca (Jujuy, Argentina). Chungara, 34 (1):35-52.

GATTO, S.

1946 Exploraciones arqueológicas en el Pucara de Volcán. Extracto de la Revista del Museo de La Plata, tomo IV: 5-91.

HASSIG, R.

1985 Trade, Tribute and Transportation: The Sixteeth-Century Political Economy of the Valley of Mexico. Norman: University of Oklahoma Press.

HODDER, I.

1982 Symbols in Action. Cambridge: Cambridge University Press.

LUTTWAK, E. N.

1976 The Grand Strategy of the Roman Empire from the First Century A.D. to the Third. Baltimore: John Hopkins University Press. 
MILLER, D.

1995a Consumption and Commodities. Annual Review of Anthropology, 24: 141-161.

1995b Acknowledging Consumption. Londres: Routledge.

MOORE, J.D.

1989 Pre-Hispanic Beer in Coastal Peru: Technology and Social Context of Prehistoric Production. American Anthropologist, 91 (3): 682-695.

NIELSEN, A.E.

1997 Tiempo y cultura material en la quebrada de Humahuaca (700-1650 d.C). Instituto Interdisciplinario Tilcara (FFyL - UBA). Tilcara.

2006 Plazas para los antepasados: Descentralización y poder corporativo en las formaciones politicas preincaicas de los Andes circumpuneños. Estudios Atacameños, 31: 63-89.

ORGAZ, M.; FEELY, A.; RATTO, N.

2007 La cerámica como expresión de los aspectos sociopolíticos, económicos y rituales de la ocupación Inka en la Puna de Chaschuil y en el Valle de Fiambalá (Dpto. Tinogasta, Catamarca, Argentina). In: Nielsen, A.; Rivolta, C.; Seldes, V.; Vázquez, M.; Mercolli, P. (Eds.) Procesos sociales prehispánicos en el sur andino. La vivienda, la comunidad y el territorio. Córdoba, Editorial Brujas: 207-236.
ORTIZ. M.G.; DELGADO, A.

1997 La Cerámica del Pucara de Volcán. Jujuy: Universidad Nacional de Jujuy.

RAMSEY, C.B.

2002 Oxcal 3.9. Oxford University.

SANTORO, C.; WILLIAMS, V.; VALENZUELA, D.; ROMERO, A.; STANDEN, V.G.

2010 An archaeological perspective on the Inka Provincial administration from the Southern Central Andes. In: Malpass, M.A.; Alconini, S. (Eds.) Distant Provinces in the Inka Empire: Toward a Deeper Understanding of Inka Imperialism. Iowa City, University of Iowa Press: 44-74.

SHIMADA, I.

2007 Craft Production in Complex Societies. Multicraft and producer perspectives. Salt Lake City, Foundations of Archaeological Inquiry: University of Utah Press.

WILLIAMS, V.

1991 Control estatal incaico en el Noroeste Argentino. Un caso de estudio: Potrero Chaquiago (Pcia. de Catamarca). Arqueología, 1: 75-124.

WILLIAMS, V.; VILLEGAS, M.P.;GHEGGI, M.S.; CHAPARRO, M.G.

2005 Hospitalidad e intercambio en los valles mesotermales del Noroeste Argentino. Boletín de Arqueologia, 9: 335-372. 